\title{
20. A NUMERICAL INTERPRETATION OF THE HOMOGENIZATION OF OBSERVATIONAL MATERIAL FOR ONE-APPARITION COMETS
}

\author{
G. SITA R S K I \\ Astronomical Institute, Polish Academy of Sciences, Warsaw, Poland
}

\begin{abstract}
The formulae developed by Bielicki for the objective weighting of observations are combined with an orbital improvement method in which the effects of perturbations are included in the differential coefficients. The procedure is applied to the orbit of comet $1953 \mathrm{I}$.
\end{abstract}

Production of a new catalogue of the orbits of one-apparition comets requires the recomputation of all the cometary orbits in order to make the material homogeneous. Recomputation of the orbits can be realized if the following problems are elaborated mathematically:

(1) weighting of the observations and rejection of doubtful ones (the method should be purely mathematical and quite objective);

(2) improvement of the orbital elements with allowance for all perturbations in the comet's motion and even in the differential coefficients;

(3) taking into account the nongravitational effects in the comet's motion after such effects have been detected by some mathematical process.

The first two of these problems have been solved mathematically and programmed for a digital computer. Formulae and a method for the computation of weights of individual observations, as well as a criterion for rejecting the 'erroneous' observations, have been developed by Bielicki (1972). The formulae were derived on the basis of statistics and probability theory.

Formulae for the correction of the orbital elements, including the perturbations in the differential coefficients, have been derived by the present author (Sitarski, 1971). The differential coefficients are obtained by numerical integration of the differential equation for the correction $\Delta \mathbf{r}$ to the comet's position vector:

$$
\frac{\mathrm{d}^{2} \Delta \mathbf{r}}{\mathrm{d} t^{2}}+k^{2} \frac{\Delta \mathbf{r}}{r^{3}}-3 k^{2} \frac{\mathbf{r}}{r^{5}}(\mathbf{r} \cdot \Delta \mathbf{r})=\operatorname{grad}(\operatorname{grad} \Omega \cdot \Delta \mathbf{r}),
$$

where $k$ is the Gaussian constant and $\Omega$ the disturbing function. Substituting

$$
\Delta \mathbf{r}=\sum_{i=1}^{6} C_{i} \mathbf{G}_{i}
$$

where the $C_{i}$ are arbitrary constants, we transform the differential equation for $\Delta \mathbf{r}$ into six differential equations for the $\mathbf{G}_{i}$. The numerical integration of these equations gives the values of the differential coefficients in the observational equations, from which the constants $C_{i}$ are obtained by the method of least squares. The appropriate 
TABLE I

Residuals for Comet Harrington 1953 I (Epoch 1953 Jan. 23.0 ET, Equinox 1950.0)

\begin{tabular}{|c|c|c|c|c|c|c|c|c|}
\hline \multirow[t]{2}{*}{ No. } & \multirow[t]{2}{*}{$t_{i}-t_{1}$} & \multicolumn{2}{|l|}{ Initial } & \multicolumn{2}{|c|}{ Without weights } & \multicolumn{2}{|c|}{ After weighting } & \multirow[t]{2}{*}{$\sqrt{ } w$} \\
\hline & & $\Delta \alpha \cos \delta$ & $\Delta \delta$ & $\Delta \alpha \cos \delta$ & $\Delta \delta$ & $\overline{\Delta \alpha \cos \delta}$ & $\Delta \delta$ & \\
\hline 1 & 0.318 & $-33 " 62$ & $+126^{\prime \prime} 78^{*}$ & $-34^{\prime \prime} 00^{*}$ & $+126^{\prime \prime} 73^{*}$ & $-33^{\prime \prime} 27^{*}$ & $+126^{\prime \prime} 51^{*}$ & 3.17 \\
\hline 2 & 2.400 & $+\quad 0.32$ & -0.48 & $-\quad 0.05$ & $-\quad 0.52$ & $+\quad 0.69$ & $-\quad 0.75$ & 3.17 \\
\hline 3 & 3.472 & $+\quad 0.71$ & $+\quad 0.38$ & $+\quad 0.34$ & $+\quad 0.34$ & +1.08 & +0.11 & 3.17 \\
\hline 4 & 3.487 & +1.45 & +0.06 & +1.09 & $+\quad 0.02$ & +1.83 & $-\quad 0.21$ & 3.17 \\
\hline 5 & 4.949 & -33.91 & $-\quad 2.40$ & $-34.27^{*}$ & $-\quad 2.44$ & $-33.52^{*}$ & $-\quad 2.67$ & 3.17 \\
\hline 6 & 6.837 & $-\quad 1.42$ & $-\quad 5.21$ & -1.76 & $-\quad 5.24$ & $-\quad 1.01$ & $-5.48^{*}$ & 3.17 \\
\hline 7 & 7.826 & $+\quad 0.42$ & $+\quad 1.09$ & $+\quad 0.08$ & +1.06 & $+\quad 0.84$ & $+\quad 0.82$ & 3.17 \\
\hline 8 & 8.793 & $+\quad 3.84$ & +1.10 & +3.51 & $+\quad 1.07$ & $+4.27^{*}$ & $+\quad 0.83$ & 3.17 \\
\hline 9 & 10.348 & $-\quad 0.18$ & $-\quad 0.56$ & $-\quad 0.51$ & $-\quad 0.59$ & +0.26 & $-\quad 0.83$ & 3.17 \\
\hline 10 & 10.374 & $-\quad 0.30$ & +1.51 & $-\quad 0.62$ & +1.49 & $+\quad 0.15$ & $+\quad 1.24$ & 3.17 \\
\hline 11 & 21.152 & $+\quad 0.46$ & $+\quad 1.00$ & $+\quad 0.20$ & $+\quad 0.98$ & +1.03 & $+\quad 0.74$ & 3.17 \\
\hline 12 & 21.185 & $+\quad 0.60$ & +1.11 & $+\quad 0.33$ & $+\quad 1.09$ & +1.16 & $+\quad 0.84$ & 3.17 \\
\hline 13 & 21.720 & -1.46 & $+\quad 2.48$ & $-\quad 1.72$ & +2.46 & $-\quad 0.89$ & +2.21 & 3.17 \\
\hline 14 & 23.746 & $-\quad 0.29$ & +0.56 & $-\quad 0.54$ & $+\quad 0.54$ & $+\quad 0.31$ & $+\quad 0.30$ & 3.17 \\
\hline 15 & 24.770 & $-\quad 3.54$ & $-\quad 0.67$ & $-\quad 3.78$ & $-\quad 0.69$ & $-\quad 2.92$ & $-\quad 0.93$ & 3.17 \\
\hline 16 & 32.864 & -3.71 & $-\quad 2.52$ & $-\quad 3.89$ & $-\quad 2.54$ & $-\quad 2.98$ & $-\quad 2.76$ & 3.30 \\
\hline 17 & 35.735 & -1.63 & -4.66 & -1.79 & -4.69 & $-\quad 0.86$ & $-\quad 4.89^{*}$ & 3.72 \\
\hline 18 & 53.903 & +1.46 & +32.96 & +1.50 & $+32.89^{*}$ & +2.51 & $+32.79^{*}$ & 0.96 \\
\hline 19 & 58.901 & +2.36 & $-\quad 0.20$ & $+\quad 2.47$ & $-\quad 0.30$ & +3.49 & $-\quad 0.36$ & 0.93 \\
\hline 20 & 59.829 & $-\quad 1.48$ & +5.41 & -1.36 & +5.31 & $-\quad 0.33$ & +5.25 & 0.94 \\
\hline 21 & 63.794 & $-\quad 0.88$ & +6.47 & - 0.69 & +6.34 & $+\quad 0.34$ & +6.31 & 1.00 \\
\hline 22 & 72.648 & +1.10 & $-\quad 1.54$ & +1.45 & -1.76 & +2.46 & $-\quad 1.72$ & 0.98 \\
\hline 23 & 81.494 & $-\quad 0.40$ & $-\quad 3.25$ & $+\quad 0.13$ & $-\quad 3.58$ & $+\quad 1.08$ & $-\quad 3.48$ & 0.93 \\
\hline 24 & 82.477 & +20.00 & $+\quad 0.84$ & $+20.56^{*}$ & +0.51 & $+21.50^{*}$ & $+\quad 0.62$ & 0.93 \\
\hline 25 & 84.441 & +2.26 & +8.53 & +2.86 & +8.17 & $+\quad 3.78$ & +8.29 & 0.90 \\
\hline 26 & 85.648 & $-\quad 0.03$ & $-\quad 5.17$ & $+\quad 0.60$ & $-\quad 5.55$ & +1.50 & $-\quad 5.42$ & 0.88 \\
\hline 27 & 86.639 & $-\quad 8.08$ & +3.26 & -7.43 & $+\quad 2.87$ & $-\quad 6.54$ & +3.01 & 0.87 \\
\hline 28 & 87.694 & $+\quad 0.24$ & -0.18 & $+\quad 0.90$ & - 0.59 & $+\quad 1.78$ & $-\quad 0.45$ & 0.86 \\
\hline 29 & 88.724 & +1.19 & $-\quad 0.36$ & $+\quad 1.87$ & $-\quad 0.78$ & $+\quad 2.74$ & $-\quad 0.63$ & 0.86 \\
\hline 30 & 89.660 & +1.88 & $+\quad 0.20$ & +2.58 & $-\quad 0.22$ & +3.44 & $-\quad 0.07$ & 0.86 \\
\hline 31 & 90.693 & +3.90 & $+\quad 0.60$ & +4.62 & +0.16 & +5.46 & $+\quad 0.32$ & 0.86 \\
\hline 32 & 91.527 & $-\quad 0.66$ & +5.81 & +0.08 & +5.36 & $+\quad 0.91$ & $+\quad 5.52$ & 0.86 \\
\hline 33 & 91.756 & +3.48 & +29.14 & +4.22 & $+28.69^{*}$ & $+\quad 5.05$ & $+28.85^{*}$ & 0.87 \\
\hline 34 & 92.524 & +7.22 & $+\quad 0.45$ & $+\quad 7.98$ & $-\quad 0.01$ & +8.79 & +0.16 & 0.87 \\
\hline 35 & 95.527 & $+\quad 2.71$ & $-\quad 1.25$ & $+\quad 3.52$ & $-\quad 1.74$ & +4.30 & -1.56 & 0.82 \\
\hline 36 & 98.655 & -6.54 & $-\quad 3.40$ & $-\quad 5.67$ & $-\quad 3.91$ & -4.95 & $-\quad 3.73$ & 0.78 \\
\hline 371 & 108.755 & $-207.04^{*}$ & -21.09 & $-206.03 *$ & $-21.63^{*}$ & $-205.48^{*}$ & $-21.45^{*}$ & 1.50 \\
\hline 381 & 109.405 & $-\quad 1.15$ & $+\quad 0.98$ & $-\quad 0.13$ & $+\quad 0.44$ & $+\quad 0.41$ & $+\quad 0.62$ & 1.40 \\
\hline 391 & 109.713 & $-\quad 0.59$ & $-\quad 0.25$ & $+\quad 0.43$ & $-\quad 0.80$ & $+\quad 0.97$ & $-\quad 0.62$ & 1.36 \\
\hline 401 & 113.722 & -1.67 & -13.54 & $-\quad 0.60$ & $-14.08^{*}$ & $-\quad 0.13$ & $-13.90^{*}$ & 1.07 \\
\hline 41 & 118.619 & -1.93 & $+\quad 2.15$ & $-\quad 0.82$ & +1.63 & $-\quad 0.43$ & $+\quad 1.79$ & 0.99 \\
\hline 421 & 118.706 & $+\quad 0.94$ & $+\quad 0.05$ & $+\quad 2.05$ & $-\quad 0.47$ & $+\quad 2.43$ & $-\quad 0.31$ & 0.99 \\
\hline 43 & 120.587 & $-133.91^{*}$ & $-121.22^{*}$ & $-132.79^{*}$ & $-121.74^{*}$ & $-132.43^{*}$ & $-121.58^{*}$ & 0.95 \\
\hline 44 & 121.742 & -11.14 & $-\quad 0.53$ & -10.01 & $-\quad 1.03$ & -9.67 & $-\quad 0.89$ & 0.95 \\
\hline 45 & 122.478 & $-\quad 2.39$ & $-\quad 1.53$ & -1.25 & $-\quad 2.03$ & $-\quad 0.93$ & $-\quad 1.88$ & 0.95 \\
\hline 46 & 123.587 & --15.07 & $-272.03^{*}$ & $-13.93^{*}$ & $-272.52^{*}$ & $-13.62^{*}$ & $-272.38^{*}$ & 0.98 \\
\hline
\end{tabular}


TABLE I (continued)

\begin{tabular}{|c|c|c|c|c|c|c|c|c|}
\hline \multirow{2}{*}{\multicolumn{2}{|c|}{ No. $t_{i}-t_{1}$}} & \multicolumn{2}{|l|}{ Initial } & \multicolumn{2}{|c|}{ Without weights } & \multicolumn{2}{|c|}{ After weighting } & \multirow[t]{2}{*}{$\sqrt{ } w$} \\
\hline & & $\overline{\Delta \alpha \cos \delta}$ & $\Delta \delta$ & $\overline{\Delta \alpha \cos \delta}$ & $\Delta \delta$ & $\overline{\Delta \alpha \cos \delta}$ & $\Delta \delta$ & \\
\hline 47 & 123.700 & $-\quad 0 ! 48$ & - $\quad 0.86$ & $+\quad 0.66$ & $-\quad 1 " 35$ & $+\quad 0 ! 97$ & $-\quad 1 " .21$ & 0.98 \\
\hline 48 & 124.587 & $+158.98^{*}$ & $-367.63^{*}$ & $+160.13^{*}$ & $-368.11^{*}$ & $+160.43^{*}$ & $-367.98^{*}$ & 1.02 \\
\hline 49 & 125.700 & -1.35 & -1.75 & $-\quad 0.20$ & $-\quad 2.23$ & $+\quad 0.08$ & $-\quad 2.10$ & 1.06 \\
\hline 50 & 126.597 & $-\quad 3.75$ & $-\quad 0.74$ & $-\quad 2.59$ & $-\quad 1.21$ & $-\quad 2.32$ & $-\quad 1.08$ & 1.06 \\
\hline 51 & 136.636 & $+\quad 2.52$ & $-\quad 0.02$ & +3.70 & $-\quad 0.35$ & $+\quad 3.84$ & $-\quad 0.27$ & 0.70 \\
\hline 52 & 138.685 & -10.79 & -22.18 & -9.61 & $-22.53^{*}$ & - 9.49 & $-22.46^{*}$ & 0.79 \\
\hline 53 & 140.684 & -9.67 & +1.08 & -8.48 & $+\quad 0.75$ & -8.39 & $+\quad 0.81$ & 0.87 \\
\hline 54 & 141.623 & -15.92 & +8.63 & $-14.73^{*}$ & +8.31 & $-14.66^{*}$ & +8.36 & 0.89 \\
\hline 55 & 143.496 & $-\quad 4.57$ & +0.13 & $-\quad 3.38$ & $-\quad 0.16$ & $-\quad 3.32$ & $-\quad 0.12$ & 0.90 \\
\hline 56 & 144.682 & $-\quad 0.53$ & -1.56 & $+\quad 0.66$ & $-\quad 1.84$ & $+\quad 0.70$ & -1.80 & 0.85 \\
\hline 57 & 146.609 & $-\quad 1.15$ & $-\quad 3.95$ & $+\quad 0.04$ & -4.21 & $+\quad 0.06$ & -4.19 & 0.79 \\
\hline 58 & 147.597 & -7.23 & -2.75 & -6.04 & $-\quad 3.00$ & -6.03 & $-\quad 2.98$ & 0.75 \\
\hline 59 & 149.737 & $-294.35^{*}$ & $-266.95^{*}$ & $-293.17^{*}$ & $-267.17^{*}$ & $-293.17^{*}$ & $-267.17^{*}$ & 0.72 \\
\hline 60 & 150.634 & $+175.88^{*}$ & $-153.86^{*}$ & $+177.06^{*}$ & -154 & $+177.04^{*}$ & $-154.07^{*}$ & 0.72 \\
\hline 61 & 150 & $-219.17^{*}$ & +10 & -217 & +10. & $-218.01^{*}$ & +10.18 & 0.72 \\
\hline 62 & 152.613 & $-\quad 0.51$ & -0.51 & $+\quad 0.67$ & $-\quad 0.71$ & $+\quad 0.64$ & $-\quad 0.72$ & 0.75 \\
\hline 63 & 153.462 & +19.37 & -7.79 & $+20.55^{*}$ & -7.98 & $+20.51^{*}$ & - 7.99 & 0.78 \\
\hline 64 & 153.770 & -1.68 & -8.28 & $-\quad 0.50$ & -8.46 & $-\quad 0.54$ & -8.48 & 0.79 \\
\hline 65 & 168.631 & $-\quad 0.30$ & $+\quad 2.08$ & $+\quad 0.84$ & $+\quad 2.07$ & $+\quad 0.68$ & $+\quad 1.98$ & 0.86 \\
\hline 66 & 168.658 & $-\quad 3.97$ & +35.39 & $-\quad 2.83$ & $+35.38^{*}$ & $-\quad 2.99$ & $+35.29^{*}$ & 0.86 \\
\hline 67 & 169.638 & +4.02 & $-\quad 0.52$ & $+\quad 5.15$ & $-\quad 0.52$ & $+\quad 4.99$ & $-\quad 0.62$ & 0.84 \\
\hline 68 & 170.630 & $+\quad 0.54$ & $+\quad 1.89$ & +1.68 & +1.90 & +1.50 & +1.80 & 0.82 \\
\hline 69 & 174.432 & $+\quad 3.98$ & $-\quad 1.16$ & $+\quad 5.10$ & $-\quad 1.11$ & $+\quad 4.90$ & $-\quad 1.23$ & 0.82 \\
\hline 70 & 174.651 & $+\quad 1.19$ & $+\quad 2.17$ & +2.31 & +2.23 & $+\quad 2.11$ & +2.10 & 0.82 \\
\hline 71 & 174.661 & $+\quad 3.19$ & +4.10 & +4.31 & +4.16 & +4.11 & +4.03 & 0.82 \\
\hline 72 & 178.659 & $+\quad 0.72$ & $-\quad 1.00$ & $+\quad 1.82$ & $-\quad 0.90$ & +1.59 & -1.05 & 0.74 \\
\hline 73 & 180.671 & $+\quad 0.28$ & $+\quad 1.28$ & +1.37 & +1.40 & +1.13 & +1.25 & 0.70 \\
\hline 74 & 181.437 & +1.85 & +3.48 & +2.93 & +3.60 & +2.69 & +3.45 & 0.70 \\
\hline 75 & 181.669 & +6.43 & $-\quad 1.54$ & +7.51 & $-\quad 1.41$ & $+\quad 7.27$ & $-\quad 1.57$ & 0.70 \\
\hline 76 & 184.674 & $-\quad 3.68$ & +6.55 & $-\quad 2.61$ & $+\quad 6.71$ & $\begin{array}{l}-\quad 2.87 \\
\end{array}$ & +6.53 & 0.70 \\
\hline 77 & 199.758 & $+\quad 1.89$ & -1.13 & $+\quad 2.85$ & $-\quad 0.82$ & $+\quad 2.52$ & $-\quad 1.07$ & 0.70 \\
\hline 78 & 202.682 & -8.81 & -35.48 & $-\quad 7.87$ & $-35.15^{*}$ & -8.22 & $-35.41^{*}$ & 0.70 \\
\hline 79 & 203.678 & -22.39 & $-\quad 2.83$ & $-21.45^{*}$ & -2.49 & $-21.81^{*}$ & $-\quad 2.76$ & 0.70 \\
\hline 80 & 207.744 & +1.60 & $-\quad 0.40$ & +2.50 & $-\quad 0.02$ & $+\quad 2.13$ & $-\quad 0.31$ & 0.70 \\
\hline 81 & 214.710 & $-\quad 4.47$ & $-\quad 4.58$ & $-\quad 3.63$ & -4.14 & $-\quad 4.04$ & $-\quad 4.46$ & 0.70 \\
\hline 82 & 231.735 & $+\quad 0.05$ & $+\quad 0.47$ & $+\quad 0.70$ & $+\quad 1.02$ & $+\quad 0.22$ & $+\quad 0.62$ & 0.70 \\
\hline 83 & 236.705 & $-\quad 3.00$ & $+\quad 0.92$ & $-\quad 2.40$ & $+\quad 1.50$ & $-\quad 2.90$ & $+\quad 1.08$ & 0.70 \\
\hline
\end{tabular}

$\begin{aligned} T & =1953 \text { Jan. } 5.41783 \\ q & =1.66498366 \\ e & =0.99594159 \\ \omega & =191^{\circ} 37^{\prime} 52^{\prime \prime} 53 \\ \Omega & =2204125.32 \\ i & =590711.86 \\ 1 / a & =+0.00243752 \\ a(\mathrm{AU}) & =410.25 \\ P(\mathrm{yr}) & =8309.6\end{aligned}$

Jan. $5.41888 \pm 0.00101$ $1.66499216 \pm 0.00001564$ $0.99592389 \pm 0.00005168$ $191^{\circ} 37^{\prime} 54^{\prime \prime} 30 \pm 2^{\prime \prime} \cdot 33$ $2204125.22 \pm 1.17$

$590711.80 \pm 2.14$ $+0.00244816 \pm 0.00003106$ $408.46 \pm 5.23$ $8255.2 \pm 158.8$ 
choice of the initial data for starting the integration of the equations for $\mathbf{G}_{i}$ allows us to obtain the coefficients in the observational equations for the corrections to the conventional orbital elements: $v, q, e, \omega, \Omega, i$ ( $v$ being the true anomaly). The formulae derived for these elements appear to be independent of the kind of orbit (elliptical, hyperbolic or parabolic).

The process of improvement of the orbit is realized by the GIER computer in the following manner. At first the orbit of a comet is corrected by application of the traditional method (rejecting the obviously erroneous observations only and regarding all the observations as equivalent). Starting from these preliminary improved orbital elements the $(\mathrm{O}-\mathrm{C})$ residuals are computed, all the perturbations in the comet's motion being included; and from the unperturbed Keplerian orbit of the comet an ephemeris is computed for the moments of observation. The $(\mathrm{O}-\mathrm{C})$ residuals are added to this unperturbed ephemeris and thus 'artificial observations' are obtained to correct the Keplerian orbit of the comet.

The Keplerian orbit is corrected iteratively, the 'erroneous' observations being rejected by means of Chauvenet's criterion. The third differences of the $(\mathrm{O}-\mathrm{C})$ residuals that result from this orbit improvement then serve as a basis for weighting the observations as follows. The local mean error of one observation for the mean moment of 20 successive observations is computed using Bielicki's formulae. If the number of observations is $N$, then the mean errors are obtained for $N-20$ mean moments. Thus, we have a numerical representation of the mean error function with respect to time. This function is smoothed by applying the Woolhouse parabolic method, as modified by Bielicki. Then the mean error of each individual observation is computed, and hence the weights of the observations are calculated by Bielicki's method. The process of weighting the observations is iterated three times.

The Keplerian orbit is improved once more by means of the weighted observations, the 'erroneous' observations now being rejected according to Bielicki's criterion. Finally the orbit is corrected with the perturbations included in the differential coefficients. In this single process the weighted observations are used and the doubtful observations rejected previously are again omitted.

The method described has been applied to the improvement of the orbit of the longperiod comet 1953 I (Harrington). Table I contains the $(\mathrm{O}-\mathrm{C})$ residuals. The asterisked residuals indicate the observations rejected in the process of orbit improvement. The initial residuals were obtained after a preliminary correction of the orbital elements. It is obvious that some observations can be regarded as erroneous and therefore they were rejected at once. Then the orbit was improved, first by giving all the observations unit weight, then by assigning the 'real' weights. In both cases the observations were subjected to selection by application of Bielicki's criterion.

The last column in Table I contains the values of square roots of the weights as computed by Bielicki's method. The rapid change in the run of weights after the seventeenth observation is due to the lack of observations during the next 20 days or so (probably because of the Full Moon). We can see that the observations of the comet were more exact before than after perihelion (perihelion time falling around the middle of the table); the ratio of the weights of the observations at the beginning 
and at the end of the observation interval amounts to $20: 1$. Hence we can hardly regard all the observations as equivalent when improving the orbit.

The orbital elements and their mean errors, as resulting from the improvement of the orbit without and with weights, are given below the table. The differences of the two improved sets are small and are contained within the limits of the mean errors of the elements. The question arises as to whether the use of this rather complicated process, described above, is reasonable in practical computations. However, the mathematical selection of observations may be applied only to equivalent observations. Furthermore, the nongravitational effects in the comet's motion can be detected by analysing the $(\mathrm{O}-\mathrm{C})$ residuals of the weighted observations. Therefore, it seems that the weighting of the observations should not be neglected in the process of orbit improvement if we really want to determine the 'definitive' orbit of the comet.

\section{References}

Bielicki, M.: 1972, this Symposium, p. 112.

Sitarski, G.: 1971, Acta Astron. 21, 87. 